INTERVENTIONAL CARDIOLOGY AND SURGERY

\title{
Outcome of transcatheter closure of muscular ventricular septal defects with the Amplatzer ventricular septal defect occluder
}

\author{
B D Thanopoulos, M L Rigby
}

Heart 2005;91:513-516. doi: 10.1136/hrt.2004.035535

See end of article for authors' affiliations

Correspondence to: Dr Basil D Thanopoulos, Department of Paediatric Cardiology, Aghia Sophia Children's Hospital, Thivon and Levadias Street, Athens 115 27, Greece; vasiliosthanopoulos@ usa.net

Accepted 19 May 2004
Objectives: To present further experience and intermediate term outcome in 30 patients with single muscular ventricular septal defects (MVSDs) who underwent transcatheter closure with the Amplatzer ventricular septal defect occluder (AVSDO).

Patients and design: Thirty patients, aged 4 months to 16 years, with MVSDs underwent transcatheter closure with the AVSDO. The device consists of two low profile disks made of Nitinol wire mesh with a $7 \mathrm{~mm}$ connecting waist. The prosthesis size (waist diameter) was selected to be equal to the balloon "stretched" diameter of the defect. A 7-9 French sheath was used to deliver the AVSDO. Fluoroscopy and transoesophageal echocardiography guided the procedure.

Results: The stretched diameter of the defects ranged from 6-14 mm. The communication was completely occluded in 28 of 30 patients (93\% closure rate). One patient (a 4 month old infant) with sustained complete left bundle branch block after the procedure went on to develop complete heart block one year later. No other complications were observed during a mean follow up of 2.2 years (range 0.25-4.5 years).

Conclusions: The AVSDO is an efficient prosthesis that can be safely used in the majority of patients with a single MVSD. Further studies are required to establish long term results in a larger patient population.
$M$ uscular ventricular septal defects (MVSDs) can exist virtually anywhere in the septum. ${ }^{1}$ They can be single or multiple, ranging from extremely small ( $1-2 \mathrm{~mm}$ ) to very large (equal to or greater than the diameter of the aortic valve). Most defects warranting surgery are closed in infancy but some smaller defects initially considered not to require closure in early life require late closure if left ventricular dimension increases, although the majority of muscular defects are small and usually close spontaneously. A right sided approach is used for surgical repair. A left ventriculotomy gives excellent exposure but it can give rise to left ventricular dysfunction and should be avoided. ${ }^{2}$ Overall results of surgery are excellent in the current era, but there is an increased risk of residual defects and the need for repeat operation when compared with perimembranous defects. ${ }^{34}$ During the past few years a variety of devices have been used to close congenital or post-infraction MVSDs, but none has gained wide acceptance. ${ }^{5-11}$ Large delivery sheaths, inability to recapture, and a very high rate of residual shunting are some of the limitations of previously used devices. Recently, Thanopoulos and colleagues ${ }^{12}$ and Hijazi and associates ${ }^{13}$ reported on their preliminary experience in children with a new device, the Amplatzer ventricular septal defect occluder (AVSDO), to close MVSDs with very good results. In this report, we present further experience and intermediate term outcome in 30 patients from two institutions with MVSDs who underwent transcatheter closure with the AVSDO.

\section{PATIENTS AND METHODS}

Since June 1998, 30 patients at two institutions have undergone transcatheter closure of an MVSD with an AVSDO. Patients were selected according to a previously agreed protocol with full and informed consent. The criteria for inclusion in the study were a cardiothoracic ratio on a chest radiograph of more than $60 \%$, a systemic ventricular end diastolic dimension of greater than $95 \%$ for age, and a defect diameter greater than $4 \mathrm{~mm}$ measured by transthoracic or transoesophageal echocardiography. Patients with important malalignment of the outlet septum, overriding of the aorta or pulmonary trunk, aortic valve prolapse, a straddling atrioventricular valve, or a distance of less than $4 \mathrm{~mm}$ between the border and semilunar or atrioventricular valve were excluded. Patients who had satisfied the criteria for entry into the study then underwent cardiac catheterisation and angiography under general anaesthesia and at the same time were studied by transoesophageal echocardiography. The patients were heparinised to achieve an activated clotting time of more than 200 seconds at the time of device implantation. An arteriovenous guidewire loop was established between the femoral artery and right internal jugular vein in the majority of patients. In a patient with an anterior defect and in one with an outlet defect, however, the approach was from the femoral vein without establishing an arteriovenous loop. In each patient the defect was sized by balloon from the venous side with an end hole balloon tipped catheter or a Meditech sizing balloon (Meditech, Watertown, Massachusetts, USA) to establish the "stretched" diameter of the defect. ${ }^{12}$ The device size (waist diameter) was selected to be equal to the measured stretched diameter. In 27 patients the defect was closed by introducing the ventricular septal defect occluder through a long sheath from the internal jugular vein. In one patient closure was achieved through a retrograde arterial route and in the remaining two through a femoral venous long sheath passed from the right to the left ventricle directly (fig 1). The final positioning of the closure device was dependent on transoesophageal echocardiographic screening, used simultaneously with standard radiography (fig 2). The device was finally released only when the position seemed satisfactory and there was no interference

Abbreviations: AVSDO, Amplatzer ventricular septal defect occluder; MVSD, muscular ventricular septal defects 

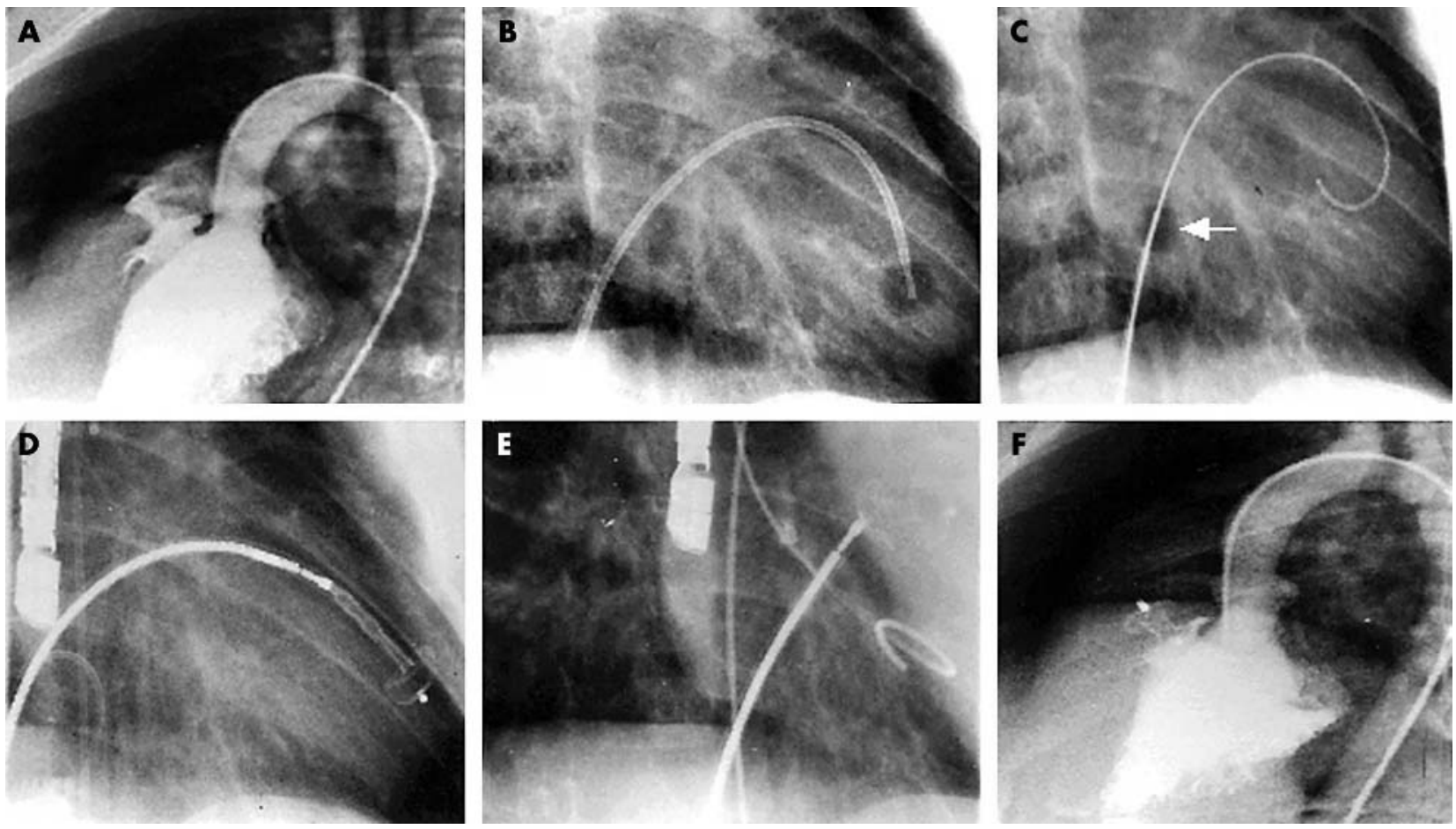

Figure 1 (A) Long axial oblique left ventriculogram showing an anterior muscular ventricular septal defect. (B) Crossing of the defect with a balloon tipped end hole catheter (right anterior oblique (RAO) $30^{\circ}$ view). (C) Advancement of an Amplatz 0.035" STl exchange wire: balloon sizing (arrow). (D) Deployment of the left ventricular disk (RAO $30^{\circ}$ view). (E) Deployment of the right ventricular disk (RAO $30^{\circ}$ view). (F) Long axial oblique left ventriculogram after release of the device showing complete closure of the defect.

with semilunar or atrioventricular valve function. Prophylactic antibiotics were not routinely given during the procedure.

All patients were discharged on the day after the procedure and prescribed aspirin 3-5 $\mathrm{mg} / \mathrm{kg}$ daily for six months. Before discharge an ECG and a 24 hour Holter ECG, a biplane chest radiograph, and a transthoracic echocardiogram were recorded. All patients had chest radiography, an ECG, and complete two dimensional and colour Doppler echocardiographic studies at 1-3 months after the procedure and then serially every six months. At 12 months' follow up examination a 24 hour Holter ECG was also recorded. Endocarditis prophylaxis was discontinued at the 12 month follow up if the defect was completely closed. A complete haemodynamic and angiographic study was scheduled for the two year follow up. Persistent shunts were angiographically and echocardiographically graded as foaming, trivial, small, moderate, and large as previously described..$^{14}$

\section{RESULTS}

Thirty infants, children, and adolescents were entered into the study. Their weights ranged from $4.2-39 \mathrm{~kg}$ (median 22 (11) $\mathrm{kg}$ ), age 4 months to 16 years (median 5.8 (3.7) years), pulmonary to systemic flow ratio $1.7-3.4$ (mean $2.4(0.3)$ ), and stretched diameter of the defect $6-14 \mathrm{~mm} \quad(8.5$ (3.2) $\mathrm{mm}$ ). Fluoroscopy and total procedural times ranged from 25-45 minutes (mean 35 minutes) and from 75-120 minutes (mean 90 minutes), respectively. The site of the ventricular septal defect varied considerably, which opened to the inlet of the right ventricle in two and was a mid-muscular defect in 16, an anterior and apical defect in seven, and an anterior and outlet defect in five. Associated abnormalities were congenitally corrected transposition of the great arteries in two, complete transposition of the great arteries after a Mustard operation in one, and a residual MVSD after complete repair of tetralogy of Fallot with pulmonary atresia.
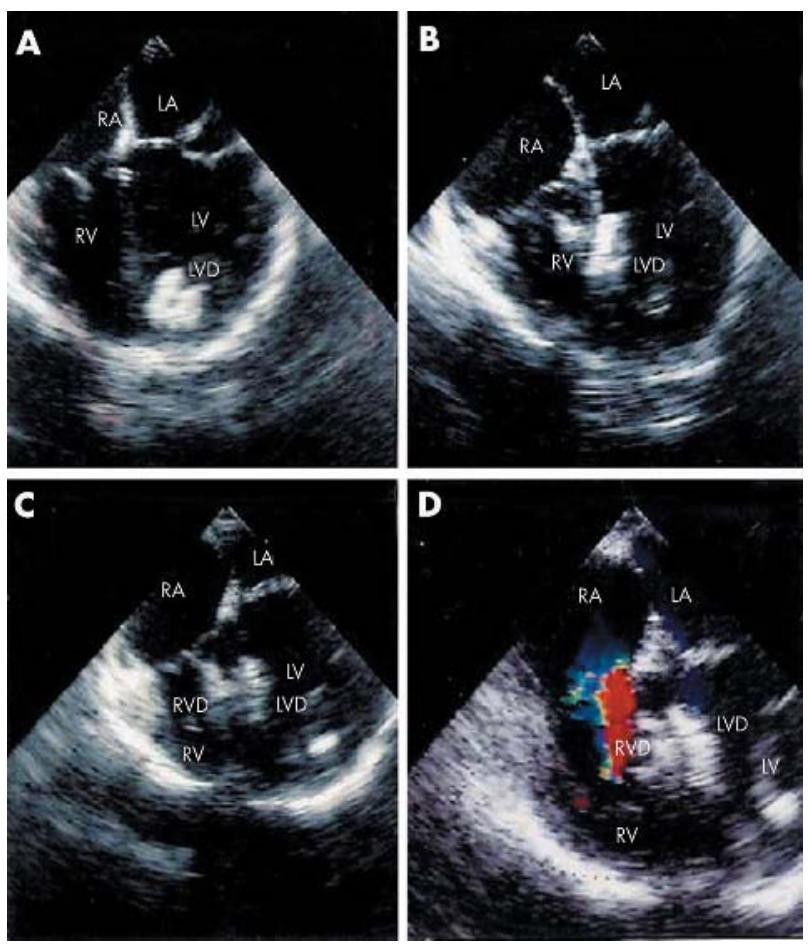

Figure 2 Transoesophageal guidance of transcatheter closure of a midmuscular ventricular septal defect with the Amplatzer ventricular septal defect occluder: four chamber view. (A) Deployment of the left ventricular disk. (B) The left disk pulled against the septum.

(C) Deployment of the right ventricular disk. (D) Colour Doppler obtained immediately after implantation of the prosthesis. Note good position of the device with no evidence of residual shunting. 
The ventricular septal defect was successfully closed in 28 of 30 patients (93\% closure rate, 95\% confidence interval $77.9 \%$ to $99.2 \%$ ). In two patients with a moderately large apical defect there was a small residual left to right shunt and one patient with a mid-muscular defect had a small additional apical defect. Four patients developed transient complete left bundle branch block at the time of the procedure, which then resolved at follow up. However, a fifth patient with sustained complete left bundle branch block and normal for age PR interval went on to develop complete heart block one year after the procedure. This was a 4 month infant who weighed $4.2 \mathrm{~kg}$ at the time of the procedure and had received an $8 \mathrm{~mm}$ occluder. In 26 patients the left ventricular end diastolic dimension, measured by transthoracic echocardiography 1-3 months after device closure, had a reduction ranging from 5-20\% (mean 9\%).No other early or late complications such as atrioventricular or semilunar valve regurgitation, device embolisation, or thromboembolic events occurred during a median follow up of 2.2 years (range $0.25-4.5$ years). Metal fatigue structures on chest radiography were not observed. Complete closure, excellent position of the device across the defect, and no evidence of device failure were observed in all 12 patients who underwent cardiac catheterisation two years after closure (fig 3).

\section{DISCUSSION}

There are few reports in the medical literature of transcatheter closure of MVSDs with the AVSDO with very good early results. ${ }^{12}{ }^{13}$ The findings of this study of a significant number of patients with a median follow up of 2.2 years indicate that transcatheter closure of MVSDs with the AVSDO can be undertaken effectively and safely in the majority of patients. The communication was completely occluded in 28 of 30 patients (93\% closure rate, $95 \%$ confidence interval $77.9 \%$ to $99.2 \%$ ) with a very low rate of significant complications (one in $30 ; 3.3 \%$ ) during the procedure or at follow up.

\section{Comparison with other occluders}

In contrast to other occluders (Rashkind or buttoned device), ${ }^{5-7}$ which were originally developed for percutaneous closure of atrial septal defects and persistent ductus arteriosus, the AVSDO was especially designed for transcatheter occlusion of MVSDs. Most important is the long connecting waist of the device, which in essence stents the communication forcing blood flow through a highly thrombogenic conduit composed of Nitinol wire mesh filled with polyester fabric. This achieves fixation and stability and results in a virtually $100 \%$ occlusion rate by thrombosis. Therefore, the AVSDO possesses small retention disks, which lower the risk of encroachment on vital cardiac structures and require a small septal rim around the defect. This unique design solved many of the limitations of previously used MVSD occluders, such as large delivery sheaths, no self centering, dislodgement and embolisation of the device, and an inability to be repositioned and redeployed..$^{5-11}$ Although we did not encounter all anatomical types of MVSDs such as multiple defects it is expected that these communications would be amenable to transcatheter closure with the AVSDO. Recently, Waight and colleagues ${ }^{15}$ reported on their preliminary experience with three patients in whom the AVSDO was used for multiple MVSDs with quite satisfactory results.

Although transoesophageal and colour Doppler echocardiography are important in monitoring transcatheter device closure of cardiac defects, in our experience for the closure of ventricular septal defects this modality should always be combined with fluoroscopic guidance. ${ }^{13}$ Repeated left ventricular injections of small amounts of contrast medium through the arterial catheter greatly facilitates the guidance of the procedure and reduces the risk of device misplacement and embolisation.

The potential complications of transcatheter closure include ventricular perforation leading to cardiac tamponade, embolisation of the device, and interference with atrioventricular valve function leading to regurgitation. It is not yet clear whether there will be a continuing risk of endocarditis. There appears also to be a risk of complete heart block after closure of a defect opening to the inlet of the right ventricle. This is not surprising because the ventricular conduction tissue axis passes above or anterosuperior to the defect ${ }^{1}$ and is overlapped by the right ventricular retention disk. The persistent motion of the heart may lead to erosion of the conduction axis, giving rise to complete atrioventricular block. As in our study this is more likely to occur in small infants who have received a relatively large device. On the basis of this experience we believe that all patients need 24 hour Holter ECG recording at regular intervals.

\section{Drawbacks}

Only three patients in the study were under 12 months of age, whereas most recent surgical series include a high proportion of infants with large defects or multiple defects presenting with heart failure and failure to thrive in early life. Although a critical appraisal of the results of this study shows that overall they are very good, they cannot be compared with most series of conventional surgery. ${ }^{3}$ It seems, however, that transcatheter device closure of a single MVSD with the AVSDO can be applied to most muscular defects and some residual defects present after cardiac surgery. It remains to be
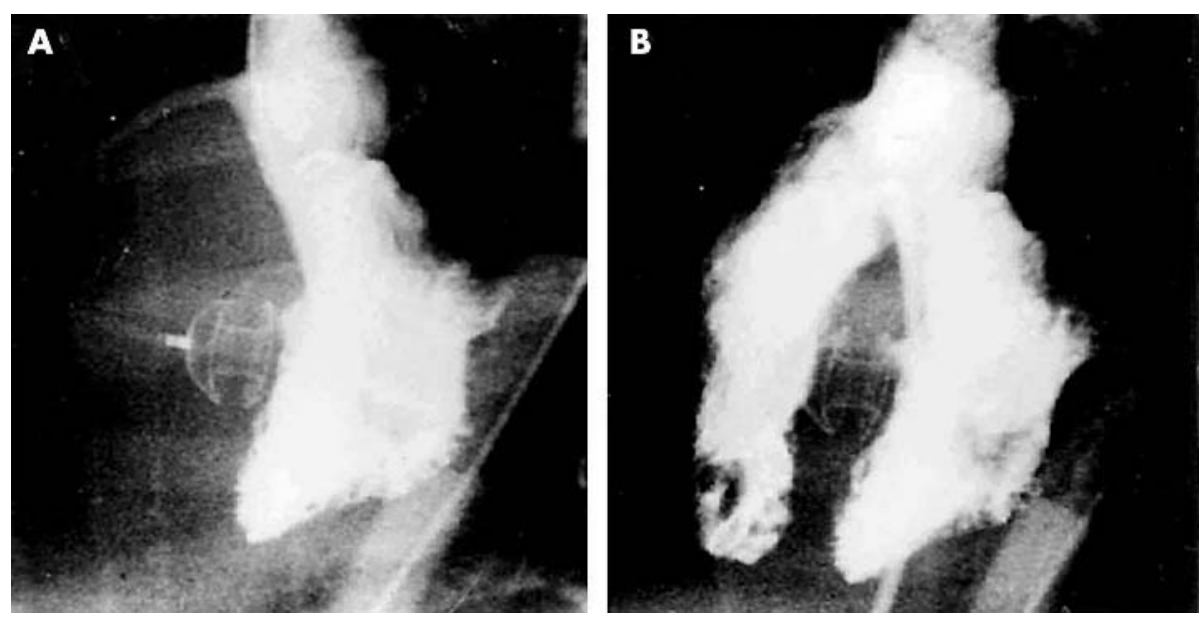

Figure 3 (A) Long axial oblique left and (B) right and left ventriculograms two years after implantation of an Amplatzer ventricular septal occluder. Note complete closure, excellent position of the device, and no evidence of device failure. 
seen whether the technique can be applied to small infants with large defects giving rise to symptoms. It is quite difficult to place the sheath through a ventricular septal defect in preparation for proper device deployment without damaging the tricuspid valve. The delivery system needs to be miniaturised to eliminate this drawback and to facilitate the use of the AVSDO in small paediatric patients. However, intraoperative closure under transoesophageal echocardiography guidance is an important alternative to device occlusion in small infants with MVSDs. ${ }^{16}$ It can also be argued that, in some of the patients in this study, the indication for closure of the defects was somewhat borderline because the defects naturally are reduced spontaneously in size and eventually close in many instances. Nevertheless, in most cases, closure of the defects leads to reduced left ventricular volume and to complete occlusion.

In summary, our results indicate that the AVSDO is an efficient prosthesis that can be safely used in the majority of patients with a single MVSD. Further studies and long term follow up are required before this technique enters into routine clinical practice.

\section{Authors' affiliations}

B D Thanopoulos, Aghia Sophia Children's Hospital, Athens, Greece M L Rigby, Royal Brompton Hospital, London, UK

There were no sources of financial support

Presented at the 52nd Annual Scientific Session of the American College of Cardiology, Chicago, 30 March to 2 April 2003.

\section{REFERENCES}

1 Anderson RH, Becker AE, Tynan M. Description of ventricular septal defects: or how long is a piece of string? Int J Cardiol 1986;13:267-78.
2 Griffiths SP, Turi GK, Ellis K, et al. Muscular ventricular septal defects repaired with left ventriculotomy. Am J Cardiol 1981;48:877-86.

3 Serraf A, Lacour-Gayet F, Bruniaux J, et al. Surgical management of isolated multiple ventricular septal defects. J Thorac Cardiovasc Surg 1992;103:437-43.

4 Kitagawa T, Durham LA, Mosca RS, et al. Techniques and results in the management of multiple ventricular septal defects. J Thorac Cardiovasc Surg 1998;115:848-56.

5 Lock JE, Block PC, McKay RG, et al. Transcatheter closure of ventricular septal defects. Circulation 1988;78:361-8.

6 Bridges ND, Perry SB, Keane JF, et al. Preoperative transcatheter closure of congenital muscular ventricular septal defects. N Engl J Med 1991;324:1312-7

7 O'Laughlin MP, Mullins CE. Transcatheter occlusion of ventricular septal defect. Cathet Cardiovasc Diagn 1989;17:175-9.

8 Nykanen DG, Perry SB, Keane JF, et al. Transcatheter occlusion of ventricular septal defects: experience in 80 patients with congenital heart disease. Circulation 1993;88:1532.

9 Rigby ML, Redington AN. Primary transcatheter umbrella closure of perimembranous ventricular septal defect. Br Heart J 1994;72:368-71.

10 Sideris EB, Walsh KP, Haddad JL, et al. Occlusion of congenital ventricular defects by the buttoned device. Heart 1997;77:276-9.

11 Janorkar S, Goh T, Wilkinson J. Transcatheter closure of ventricular septal defects using the Rashkind device: initial experience. Cathet Cardiovasc Diagn 1999;46:43-8.

12 Thanopoulos BD, Tsaousis GS, Konstadopoulou GN, et al. Transcatheter closure of muscular ventricular septal defects with the Amplatzer ventricular septal defect occluder: initial clinical applications in children. J Am Coll Cardiol 1999;33:1395-9.

13 Hijazi ZM, Hakim F, Al-Fadley F, et al. Transcatheter closure of single muscular ventricular septal defects using the Amplatzer muscular VSD occluder: initial results and technical considerations. Catheter Cardiovasc Interv 2000;49:167-70.

14 Thanopoulos BD, Laskari CV, Tsaousis GS, et al. Closure of atrial septal defects with the Amplatzer occlusion device: preliminary results. J Am Coll Cardiol 1998;31:1110-6.

15 Waight DJ, Bacha EA, Kahana M, et al. Catheter therapy of Swiss cheese ventricular septal defects using the Amplatzer muscular VSD occluder. Catheter Cardiovasc Interv 2002;55:355-61.

16 Amin Z, Berry LM, Foker JE, et al. Intraoperative closure of muscular ventricular septal defect in a canine model and application of the technique in a baby. J Thorac Cardiovasc Surg 1998;115:1374-6.

\section{IMAGES IN CARDIOLOGY}

\section{Janeway lesions in infective endocarditis}

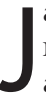
aneway lesions are one of the stigmata of infectious endocarditis. They are irregular, erythematous, flat, painless macules on the palms, soles, thenar and hypothenar eminences of the hands, tips of the fingers, and plantar surfaces of the toes; they rarely present as a diffuse rash, and are very rare in clinical practice.

A 25 year old woman presented with prolonged fever. She was on regular penicillin prophylaxis for rheumatic mitral valve disease. She had fever at the time of presentation. Her physical examination revealed erythematous macular painless rashes distributed along the thenar and hypothenar eminences of both hands (panel A), which were more pronounced on the left hand (panel B). She had subconjunctival haemorrhages and subungual splinter haemorrhages. Cardiac examination revealed moderate mitral regurgitation and mild mitral stenosis. She also had hepatosplenomegaly.

The diagnosis of infective endocarditis was confirmed by blood cultures,
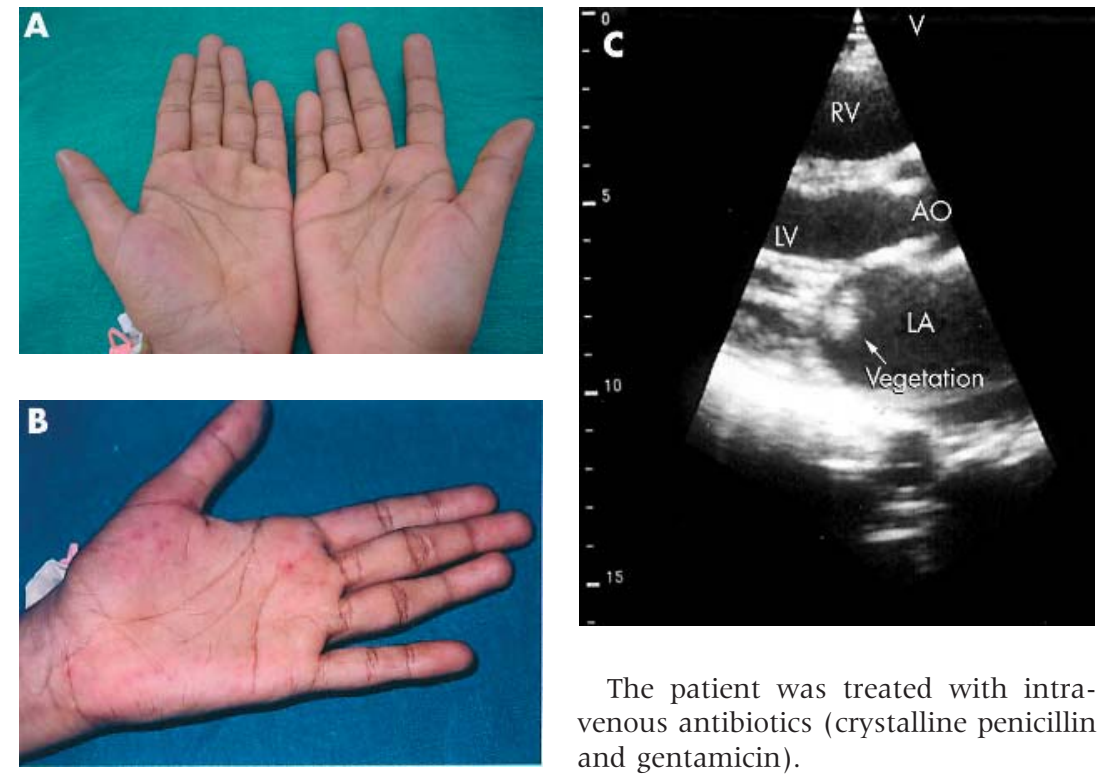

The patient was treated with intravenous antibiotics (crystalline penicillin and gentamicin).

which grew Streptococcus viridans in three separate cultures, and echocardiographic demonstration of vegetation attached to the mitral valve (panel C).
S M Divakaramenon

R Krishnan R Chandni mdsmenon@hotmail.com 